\title{
FACTORES QUE AFECTAN EL INTERVALO PARTO PRIMER SERVICIO EN VACAS LECHERAS DE CRIANZA INTENSIVA
}

\author{
Roberto Evaristo $\mathbf{R}^{1}$ y Luisa Echevarría C. ${ }^{2}$
}

\section{Alstract}

Calving - first service (IPPS) and calving - ovulation (IPPO) intervals were determined in 128 dairy cows from 5 intensively managed farms in Lima, Peru. Progesterone levels, determined by radioinmunoassay in a total of 1450 skim milk samples taken twice weekly from 15 days post partum until first artificial insemination, were used to determine IPPO. Field data on calving number (primiparous and multiparous), calving season (Spring September-December and Summer JanuaryApril), body condition (BCS evaluated monthly starting 30 days post partum) and milk production were collected. Multiple regression analysis (GLM), with IPPO and IPPS as dependent variables, and calving season, calving number, changes in BCS, milk production, heat detection efficiency and farm as independent variables, revealed an IPPO of $45+19.9$ days and IPPS of $99.2+50.1$ days. IPPO was influenced by calving season and farm, and IPPS by heat detection efficiency, BCS at calving and farm.

Key words: Calving season, reproductive parameters, ovarian activity, body condition.

\section{Resumen}

Se estudiaron 128 vacas lecheras pertenecientes a 5 establos de Lima con un sistema de manejo intensivo. Se determinó los siguientes parámetros reproductivos: Intervalo Parto Primer Servicio (IPPS) e Intervalo Parto Primera Ovulación (IPPO). El IPPO fue obtenido por niveles de progesterona de leche descremada mediante la técnica del Radio Inmuno Análisis (RIA). La frecuencia del muestreo se hizo dos veces por semana desde el quinceavo día postparto hasta la primera inseminación Artificial (IA). Se analizaron 1450 muestras de leche. Los datos de campo fueron recolectados de los establos y fueron: Número de parto (Primíparas y Multíparas), estación de parto, (primavera: setiembre - diciembre; verano: enero - abril), condición corporal y producción de leche. La condición corporal se tomo al parto y cada 30 días hasta el servicio. El análisis estadístico consistió en regresión múltiple (GLM), donde las variables dependientes fueron: IPPO e IPPS y las variables independientes: Estación de parto, número de parto, condición corporal, producción de leche, eficiencia de detección de celo y establo. Los resultados indican un IPPO de $45 \pm 19.9$ días y un IPPS de 99.2 \pm 50.1 días. Factores con influencia significativa en IPPO fueron estación de parto y establo y en IPPS, eficiencia de la detección de celo, condición corporal al parto y establo.

Palabras clave: Estación de parto, parámetros reproductivos, actividad ovárica, condición corporal. 


\section{Introinecion}

El comportamiento reproductivo tiene una gran importancia debido a que es uno de los componentes para conseguir mayor productividad animal. En consecuencia, un entendimiento adecuado de los mecanismos que gobiernan la función reproductiva y de los factores medio ambientales que los afectan resulta indispensable para hacer correctivos tendientes a mejorar la eficiencia reproductiva. Se sabe que en el ganado lechero europeo el ambiente afecta la reproducción a través de la temperatura ambiental, debido a que esta clase de animales tiene su temperatura de confort alrededor de 13 a $18^{\circ} \mathrm{C}$ (Gilad et al., 1993). En Lima, durante el verano se llega a $31^{\circ} \mathrm{C}$ y $80 \%$ de humedad relativa, lo que quiere decir que el ganado lechero en Lima tiene un índice mayor a 72 de THI (índice de temperatura ambiental y humedad relativa) (Armstrong, 1994). Esta situación causa un estrés térmico (Shearer y Beede, 1990), que origina un pobre rendimiento reproductivo en las vacas. (Hansen, 1997;
Chemineau, 1993). En efecto, aunque faltan informes cuantificados es bien conocido que en los meses de verano en comparación con los de invierno las vacas tienden a tener mayores dificultades para preñar. Para tratar de superar este problema es de interés conocer el efecto del strés de calor sobre el intervalo parto primera ovulación, eficiencia en la detección de celo y tasa de concepción y variables relacionados como cambios de condición coporal post parto y el efecto de estos sobre los intervalos parto primer servicio y parto concepción.

\section{Materiales y Métodos}

El trabajo se realizó en Lima situada en la costa central del Perú, con una temperatura ambiental que varía durante el año desde $12-16^{\circ} \mathrm{C}$ en el invierno a $25-30$ ${ }^{\circ} \mathrm{C}$ en el verano. La humedad relativa está entre 85-95\% durante todo el año y tiene una precipitación pluvial de 26.6 a $29.2 \mathrm{~mm}$ (Fig. 1).

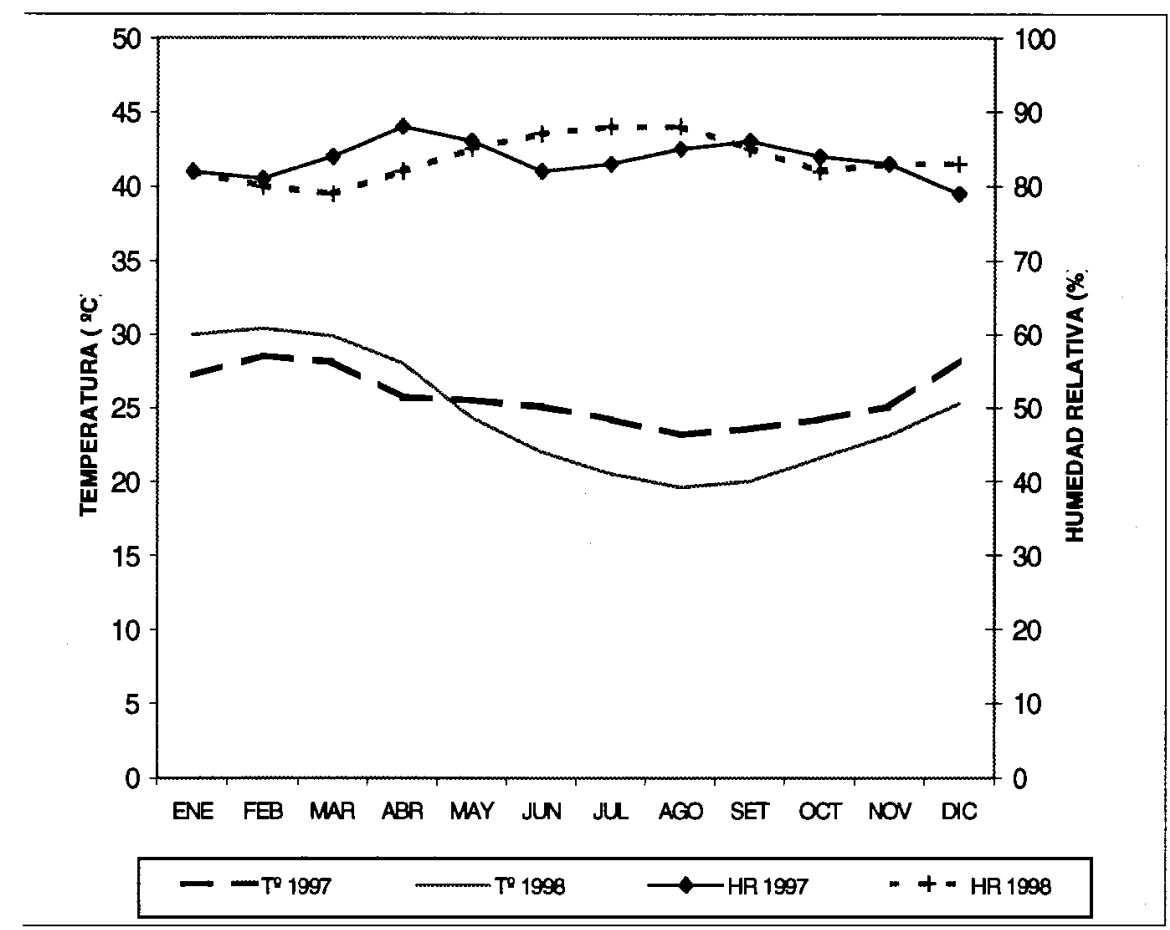

Figura 1. Temperatura $\left(\mathrm{T}^{\circ}\right)$ y humedad relativa $(\mathrm{HR})$ media mensual de Lima en los años de 1997 y 1998. 
El tamaño de los establos fluctúa entre 80 a 800 vacas bajo crianza intensiva, con un tamaño de finca de 2.5 a 120 ha. La alimentación es a base de pasto cortado y concentrados. El ordeño se realiza dos veces al día. Algunos establos procesan la leche y envían al mercado productos elaborados como yogur y queso.

La inseminación artificial se realiza mayormente con semen importado y cada establo tiene su propio técnico inseminador, la detección de celo se hace dos veces al día.

Se usaron 128 vacas lecheras para monitorear la actividad ovárica post parto a través de determinaciones de progesterona en muestras de leche. Las vacas fueron seleccionadas por estación de parto (G1: Primavera, setiembre - noviembre y G2: Verano, diciembre - marzo). Se registraron: Condición corporal al parto y cada 30 días usando la escala de 1 al 5 (Ferguson et al., 1994), Producción de leche mensual y manifestaciones de celo. La colección de muestras fue dos veces a la semana a partir del quinceavo día post parto hasta el primer servicio.

La eficiencia de la detección de celo fue calculada a partir de los celos detectados por el personal del establo y el número de ovulaciones detectadas a través de los análisis de progesterona. El análisis de los intervalos reproductivos fue hecho por regresión múltiple, considerando como variables dependientes: Intervalo Parto Primera Ovulación (IPPO), con número de parto, estación de parto, Cambios de condición corporal (CC) post parto y establo como variables independientes; Intervalo Parto Primer Servicio (IPPS) con número de parto, estación de parto, Cambios de CC post parto, eficiencia detección de celo y establos, como variables independientes; e Intervalo Parto Concepción (IPC) con factores de variación como: establo, número de parto, IPPS y estación de parto. Los cambios de CC y la producción de leche fueron sometidos a análisis de variancia con muestras repetidas en el tiempo, considerando factores de variación: estación y número de parto.
Los niveles de progesterona fueron determinados por Radioinmunoensayo usando el Kit FAO/IAEA ${ }^{125}$ I para progestrona de Fase sólida (Nachreiner $e t$ al., 1986). Los coeficientes de variación intra en inter ensayo de los controles de calidad internos bajos y altos fueron: 12.5 , $17.4 \%$ y $10.24,18.79 \%$, respectivamente.

\section{Resultados}

El Intervalo Parto Primera Ovulación (IPPO) fue $45 \pm 19.9$ días ( $n=65$ ), y fue influenciado de modo significativo por $\mathrm{CC}$ al parto, estación de parto y establo. Las vacas que parieron durante la primavera tuvieron un IPPO de $42.8 \pm 2.6$ días, y las paridas en verano $23.2 \pm 6.1$ días.

La eficiencia en la detección de celo determinada en cinco establos fue de: 58 , $38,51,26$ and $0 \%$ para los establos, $1(n=29)$, $2(n=18), 3(n=18), 4(n=11)$, y $5(n=4)$, respectivamente.

El IPPS global fue de $99.2 \pm 50.1$ días $(\mathrm{n}=121)$ y los factores con influencia significativa fueron: Eficiencia en la detección de celo, Cambios de CC post parto y establo.

El IPC global fue de $224.6 \pm 85$ y fue afectado por la producción de leche al primer mes, establo, IPPS y cambios de la CC del parto a los 60 días

La Producción de leche y los cambios de CC postparto fueron afectados por estación de parto $(\mathrm{P}<0.01)$ (Fig. 2).

\section{Discusión}

El IPPO encontrado para las vacas paridas en primavera fue más largo que el reportado en la literatura (Lucy, et al.,1992), la explicación estaría dada por el resultado que da el análisis de los cambios de CC post parto, donde hubo un efecto significativo de la estación de parto, y es así que estas vacas 


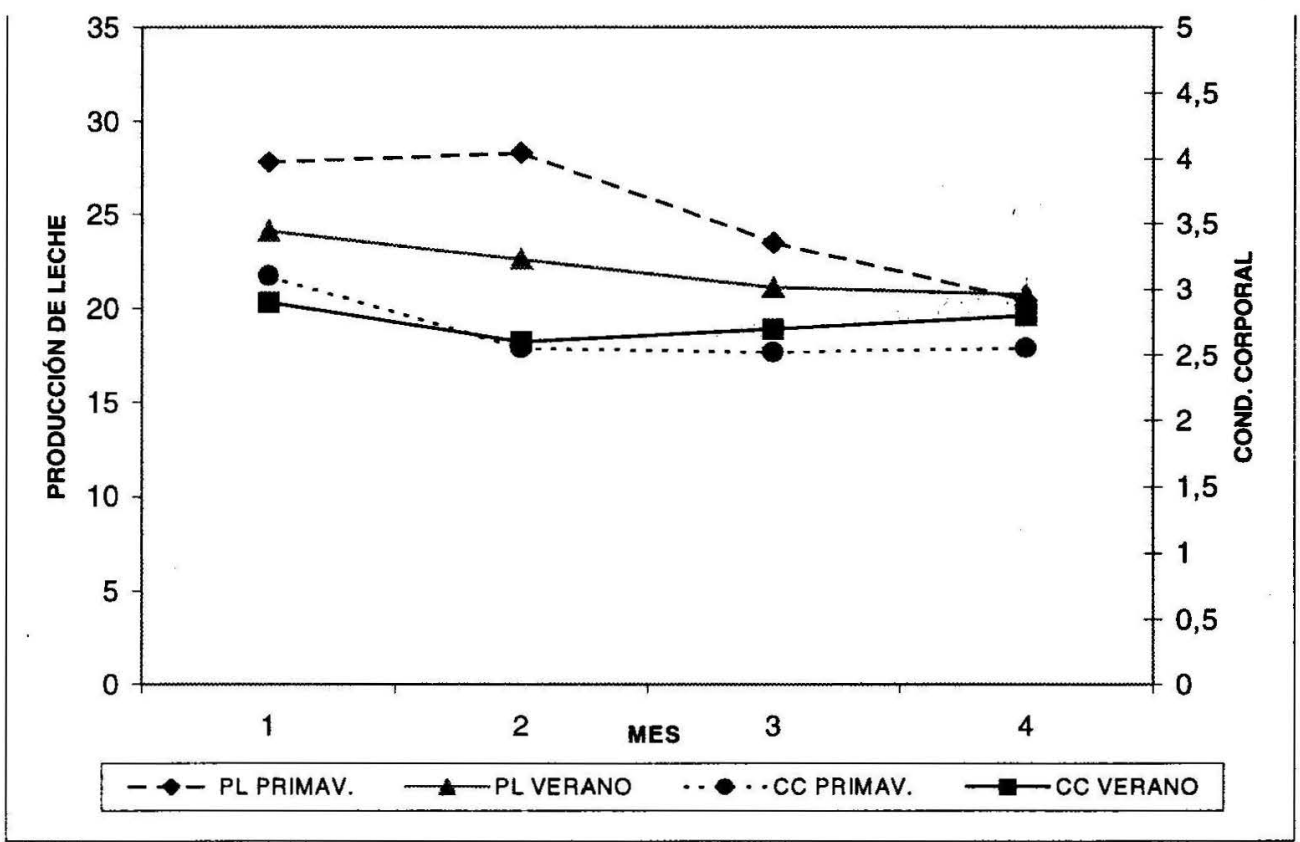

Figura 2. Relación entre la producción de leche (PL) y condición corporal (CC) durante la primavera y verano.

pierden mayor $\mathrm{CC}$ debido a la mayor producción de leche que tienen desde el inicio de la lactación (Fig. 2), no pudiendo recuperar CC tan rápido como lo hacen las vacas paridas en verano. El balance energético negativo (BEN) origina una hipoglicemia, que incrementa la liberación de la Hormona de Liberación Corticotropina (CRH), que a su vez estimula la liberación de ACTH y B endorfina ( Thun et al., 1996), que afectan negativamente la pulsatilidad de GnRH y la secreción de LH, y por lo tanto el inicio de la actividad ovárica (Gilad et al.,1993).

Las vacas paridas en verano tuvieron un IPPO más corto, y a su vez la producción de leche más baja y perdieron menor $\mathrm{CC}$ al inicio de la lactación (Fig 2) que las de primavera, por lo que se asume que habrían tenido un menor balance energético negativo.

Se deduciría que, para que en las vacas post parto ocurra la primera ovulación sería más importante, el grado de balance enrgético negativo que alcancen al inicio de la lactación. (Confield y Butler, 1991;
Schillo, 1992), que el efecto del estrés de calor.

Otro factor de importancia para el IPPS es la eficiencia en la detección de celo, aunque se encontró una eficiencia aceptable en algunos establos, existió una alta y significativa correlación negativa entre las dos variables, por lo que establos con alta eficiencia tendrían un menor IPPS.

Con relación al IPC, los resultados corresponden a animales problema, y la explicación radicaría en que, a pesar de que las vacas habrían estado ciclando, están siendo inseminadas en un HTI $>72\left(27-32^{\circ} \mathrm{C}\right.$ y $80 \%$ de Humedad Relativa), que es considerado como estrés térmico para vacas lecheras (Armstrong, 1994) y en consecuencia existe una baja tasa de concepción. El estrés térmico afecta la tasa de concepción al producir en las vacas un patrón irregular en la secreción de estradiol o la elevación de la temperatura de ambiente uterino, que causarían alta mortalidad embrionaria temprana (Ealy et al., 1994). 
Los animales más perjudicados resultaron ser las vacas paridas en primavera, pues además de la demora en la primera ovulación su oportunidad de quedar preñadas será mayor cuando acabe el verano, y esto significa un mayor IPC en relación al resto de vacas del hato. A diferencia de las vacas paridas en verano, que ovulando más rápido preñan rápidamente a la llegada del otoño.

Existen diferentes tecnologías descritas para mejorar la tasa de concepción en condiciones de estrés térmico, así como de manejo nutricional para disminuir los cambios de condición corporal post parto en verano, pero su aplicación deberá ser sugerida después de una validación y evaluación costobeneficio.

\section{Literatura Ctada}

I. Armstrong, D.V. 1994. Heat Stress interaction with shade and cooling. J. Dairy Sci. Vol.77:2044-2050

2. Chemineau, P. 1993. Medioambiente y Reproducción Animal. Revista Mundial de Zootecnia . FAO . 77:2-14.

3. Confield, R. W. y W.R. Butler. 1991. Energy balance, first ovulation and the effects of naloxone on LH secretion in early post partum dairy cows. J. Anim. Sci. 69: 740

4. Ealy, A.D., C. Arechiga, D. Obray, D., C. Risco y P. Hansen. 1994. Effectiveness of short term cooling and Vitamin $E$ for alleviation of infertility induced by heat stress in dairy cows. J dairy Sci 77: 3601-3607

5. Ferguson, J.D., D.T. Galligan y N. Thomsen. 1994. Principal descriptors of body condition score in Holstein cows.
J.Dairy Sci. 77:2695-2703

6. Gilad, E., R. Median, A. Berman, Y. Graber y D. Wolfenson. 1993. Effect of heat stress on tonic and Gn-RH induced gonadotropin secretion in relation to concentration of oestradiol in plasma of cyclic cows. J.Reprod. and Fertility (99): 315-321

7. Hansen, P.J. 1997. Effects of Environment on Bovine Reproduction. In: Current Therapy in Large animal Theriogenology. W.B. Saunders Company. Vol.1:478pp.

8. Lucy, M.C., J. Beck, C.R. Staples, H.H. Head, R.L. De La Sota y W.W. Thatcher. 1992. Follicular dynamics, plasma metabolites, hormones and insulinlike growth factor (IGF-I) in lactating cows with positive or negative energy balance during the preovulatory period. Reprod Nutr Dev 32: 331-341.

9. Nachreiner, R.F., S.J.Oschmann, L.E. Edqvist y J.I. Richards. 1986. «Solid-phase radioinmunoassay (RIA) appropriate for use in developing countries». Nuclear and Related techniques in Animal Production and Health ( Proc Sym. Vienna, 1986) IAEA.p.653-659. Vienna.

10. Schillo, K. 1992. Effects of dietary energy on Control of Luteinizing Hormone secretion in cattle and sheep. J. Anim. Sci 70: 1271 - 1282.

11. Shearer, J.K. y D.K. Beede. 1990. Thermoregulation and physiological responses of dairy cattle in hot weather. Thermal stress. Part..1 Agri-Practice Julio/Agosto Vol.11 (4): 5-17.

12. Thun, R., C. Kaufmann, H. Binder, M. Dobelli, $H$. Kundig $y \quad T$. Scheurmann. 1996. Reprod dom anim. 31:571-574. 